\title{
Evaluation and improvement of the regulatory inference for large co-expression networks with limited sample size
}

Wenbin Guo ${ }^{1,2}$, Cristiane P. G. Calixto², Nikoleta Tzioutziou², Ping Lin ${ }^{3}$, Robbie Waugh²,4, John W. S. Brown²,4 and Runxuan Zhang ${ }^{1 *}$ (D)

\begin{abstract}
Background: Co-expression has been widely used to identify novel regulatory relationships using high throughput measurements, such as microarray and RNA-seq data. Evaluation studies on co-expression network analysis methods mostly focus on networks of small or medium size of up to a few hundred nodes. For large networks, simulated expression data usually consist of hundreds or thousands of profiles with different perturbations or knock-outs, which is uncommon in real experiments due to their cost and the amount of work required. Thus, the performances of co-expression network analysis methods on large co-expression networks consisting of a few thousand nodes, with only a small number of profiles with a single perturbation, which more accurately reflect normal experimental conditions, are generally uncharacterized and unknown.
\end{abstract}

Methods: We proposed a novel network inference methods based on Relevance Low order Partial Correlation (RLowPC). RLowPC method uses a two-step approach to select on the high-confidence edges first by reducing the search space by only picking the top ranked genes from an intial partial correlation analysis and, then computes the partial correlations in the confined search space by only removing the linear dependencies from the shared neighbours, largely ignoring the genes showing lower association.

Results: We selected six co-expression-based methods with good performance in evaluation studies from the literature: Partial correlation, PCIT, ARACNE, MRNET, MRNETB and CLR. The evaluation of these methods was carried out on simulated time-series data with various network sizes ranging from 100 to 3000 nodes. Simulation results show low precision and recall for all of the above methods for large networks with a small number of expression profiles. We improved the inference significantly by refinement of the top weighted edges in the pre-inferred partial correlation networks using RLowPC. We found improved performance by partitioning large networks into smaller co-expressed modules when assessing the method performance within these modules.

Conclusions: The evaluation results show that current methods suffer from low precision and recall for large co-expression networks where only a small number of profiles are available. The proposed RLowPC method effectively reduces the indirect edges predicted as regulatory relationships and increases the precision of top ranked predictions. Partitioning large networks into smaller highly co-expressed modules also helps to improve the performance of network inference methods.

The RLowPC R package for network construction, refinement and evaluation is available at GitHub: https://github. com/wyguo/RLowPC.

Keywords: Gene co-expression networks, Gene regulatory networks, Network method evaluation, Partial correlation, Synthetic data

\footnotetext{
*Correspondence: Runxuan.zhang@hutton.ac.uk

${ }^{1}$ Information and Computational Sciences, The James Hutton Institute,

Invergowrie, Dundee, Scotland DD2 5DA, UK

Full list of author information is available at the end of the article
} 


\section{Background}

Over the last fifteen years, there has been a growing interest in reverse engineering of Gene Regulatory Networks (GRNs) that aim to infer complex graphs representing transcriptional regulatory relationships, directly from gene expression profiles [1-15]. Due to its low computational complexity as well as lower requirements for the number of samples, co-expression network analysis has been widely used to infer gene regulatory networks from high throughput expression data, such as microarray or RNA-seq data [10, 1619]. Typically thousands of genes/transcripts of special interest (e.g. differentially expressed) are utilized to construct the co-expression network in an experiment. Top candidates whose expression correlates with the gene of interest are usually further examined to identify novel regulators/targets. Despite this approach being widely used, there is a general lack of studies on the precision (the fraction of inferred regulatory relationships that are correct) and recall (the fraction of regulatory relationships that are inferred) expected.

Considerable effort has been made to evaluate the performance and robustness of GRN inference methods. The majority of evaluations were implemented on in silico datasets simulated from reference networks with sizes up to a few hundred or 1-2000 genes. Numerous studies using a range of network sizes, time-series data and perturbations have compared different analysis methods. Results are variable in terms of the top-performing method (Summaries in Additional file 1: Table S1). A series of studies have been carried out by the Dialogue for Reverse Engineering Assessments and Methods (DREAM) project, which generates challenges and organizes contests annually. The DREAM3 challenge presents gene network inference problems based on in silico networks of sizes ranging from 10, 50 and 100 genes [20-24]. Gene expression data was simulated using these networks for the following scenarios: 1) the steady state of the unperturbed networks, as well as steady state of the network where every gene is knocked out or down; and 2) 4, 23 and 46 different time series for the size 10, 50 and 100 networks respectively, with 21 time points for each time series. For example, for the network of size 100, there are a total of 1067 gene expression profiles with different perturbations and knockout/knockdown experiments available to make the inference. The inference methods: Scan Bayesian Model Averaging (ScanBMA), Gene Network Inference with Ensemble of trees (GENIE3) and Minimum Redundancy NETworks using Backward elimination (MRNETB) were the top performers in three different studies using the DREAM4 challenge time-series data, which is composed of five perturbation experiments for size 10 networks and ten perturbation experiments for size 100 networks, each with 21 time points [24-27] (Additional file 1: Table S1). Besides the DREAM benchmark datasets, the Bayesian Network (BN), Graphical Gaussian models (GGMs) and Relevance Network (RN) methods were compared using expression simulations of 100 sample points for a size 11 network with BN and GGM performing best [12]. The Algorithm for the Reconstruction of Accurate Cellular Networks (ARACNE) method had a much better performance than $\mathrm{BN}$ and $\mathrm{RN}$ on expression data with 1000 samples simulated from size 100 networks [28] while MRNET was the top ranked method when compared to the RN, ARACNE and Context likelihood or relatedness (CLR) methods on 30 datasets with different network sizes (from 100 to 1000) and sample sizes (from 100 to 1000) [29] (Additional file 1: Table S1).

A few studies aimed to evaluate network methods on larger networks of a few thousand genes. In the DREAM5 challenge, Least Absolute Shrinkage and Selection Operator (LASSO), CLR and GENIE3 are top performers among more than 30 network inference methods on a size 1643 network with 805 simulated gene expression profiles, where a list of regulators (potential transcriptional factors) are given [30]. Ten network inference methods on size 1000 network from S. Rogers [31], size 300 and 1000 networks from SynTReN [32] and size 1565 and 2000 networks from GeneNetWeaver (GNW) [24] were assessed using simulated datasets of 1000, 800, 1000, 1565 and 2000 experiments individually. CLR, GENIE3 and MRNET were the top performers in this study [33]. Similarly, ARACNE, GeneNet, Weighted Correlation Network Analysis (WGCNA) and Sparse PArtial Correlation Estimation (SPACE) were compared using size 17, 44, 83, 231, 612 and 1344 networks over datasets with 20,50,100, 200, 500 and 1000 sample points simulated from Gaussian distribution [34]. GeneNet ranked in the first place followed by ARACNE (Additional file 1: Table S1).

Despite the large number of evaluation studies, none have explored the normal experimental situation where a regulatory network is generated which involves hundreds and thousands of genes with only a small number of profiles being available. The assessments in the literature were based on either small and medium sized networks or datasets with a large number of samples. The evaluation conclusions were also based on a large amount of simulated expression profiles which would be difficult to validate experimentally due to the prohibitive cost or the amount of work in real experiments $[35,36]$.

Distinguishing direct regulatory interactions from indirect associations has been one of the major challenges in gene regulatory network constructions [2, 21] (see Fig. 1a). Partial Correlation (PC) is one of the methods used as a solution to distinguish direct from indirect edges of each pair of 

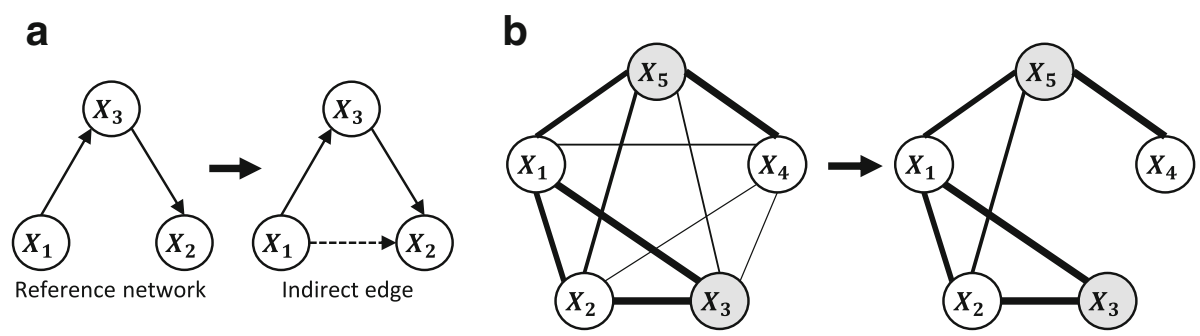

Fig. 1 Indirect edge and RLowPC network. a An indirect association from $X_{1} \rightarrow X_{2}$ could arise from a regulatory structure of $X_{1} \rightarrow X_{3} \rightarrow X_{2}$. b RLowPC network inference. In an RLowPC network, firstly only the top ranked edges are kept in a pre-inferred PC network and then for each pair of genes, only the immediate neighbours will be regressed for $\mathrm{PC}$ calculation. In this example only the top 6 of 10 edges with highest correlations are kept and PC between $X_{1}$ and $X_{2}$ is re-calculated by removing the effects from two immediate neighbouring nodes $\left(X_{3}, X_{5}\right)$. The correlation values are represented by the thickness of the edges

candidates by calculating the correlations after removing the linear dependencies from the remaining genes (see Fig. 1b). Other methods dealing with indirect connections include Partial Correlation coefficient with Information Theory (PCIT), ARACNE, MRNET and MRNETB. PCIT and ARACNE use the Information Theory of Data Processing Inequality method to remove the weakest gene association in each possible triplet structure in a network [37]. PCIT uses first order PC (removing the linear dependencies from the third gene in each possible triplet) to measure the significance of edge associations [38], whilst ARACNE uses Mutual Information (MI) to measure the associations between any two edges in each possible triplet [28]. MRNET uses a minimum redundancy feature selection method [39], where for each candidate gene in a MI network, it selects a subset of its highly relevant genes while minimising the MI between the selected genes [29]. MRNETB is an improved version of MRNET using a backward selection strategy starting from assuming that all genes are connected to the candidates. Less relevant genes are eliminated until the difference between the MI between a candidate and its neighbours and the MI within the neighbours are optimised [27].

Given that the search space for regulatory relationships expands factorially with the number of genes included in the network, the precision and recall of regulatory inference decrease with the increase of the network size. As gene clusters with highly cohesive patterns give rise to high correlations between all pairs of the genes in that cluster, the top ranked highly co-expressed genes may also be prone to errors of indirect associations. Here, we have developed a new method named Relevance Low order Partial Correlation (RLowPC), which is a refinement of top inferred edges by Partial Correlation methods. RLowPC selects top ranked edges from an inferred PC network as a reduced search space for indirect edges. We evaluated RLowPC alongside PC, PCIT, ARACNE, MRNET, MRNETB, and CLR on simulated time-series data and the summaries of the evaluated network inference methods is shown in Table 1. Precision and Area Under Precision-Recall curves (AUPR) were used as metrics to show that RLowPC outperforms the other methods.

\section{Methods}

Relevance low order partial correlation (RLowPC)

The conventional pair-wise PC measures correlations after linear dependencies on all the remaining genes are removed, the majority of which may not connect to the candidates, especially in large networks where the majority of the genes only have few linked neighbours [40, 41]. Low order partial correlation methods have been proposed and

Table 1 Summaries of the evaluated network inference methods

\begin{tabular}{|c|c|c|c|c|}
\hline Category & Methods & Cor-based & MI-based & Ref. \\
\hline \multirow[t]{6}{*}{ Deal with indirect edges explicitly } & RLowPC & Yes & & \\
\hline & PC & Yes & & {$[2,45]$} \\
\hline & PCIT & Yes & Yes & {$[33,38,50,51]$} \\
\hline & MRNET & & Yes & {$[29,33,39,50]$} \\
\hline & MRNETB & & Yes & {$[27,29,33,50]$} \\
\hline & ARACNE & & Yes & {$[28,33,50]$} \\
\hline \multirow[t]{3}{*}{ Not deal with indirect edges } & Cor & Yes & & \\
\hline & CLR & & Yes & {$[33,48,50]$} \\
\hline & Random & & & \\
\hline
\end{tabular}

Nine correlation-based, Ml-based and random network inference methods have been compared and evaluated in this study. The methods are classified into two main groups: Deal with indirect edges explicitly and Not deal with indirect edges 
utilized in the past to reduce computational complexity without much sacrifice in prediction accuracy. For example, de la Fuente et al. [42] proposed to calculate up to second order partial correlations regressing against all the remaining genes. This method was improved by confining the second order partial correlation calculation only in cases where both zero and one order PC are non-zero [43]. Our proposed
RLowPC method, firstly, reduces the search space by only picking the top ranked genes from partial correlation analysis and, secondly, computes the PC by only removing the linear dependencies from the shared neighbours in the confined search space, largely ignoring the genes showing lower association and which are less relevant in the pair-wise PC calculation. The implementation details are shown below:

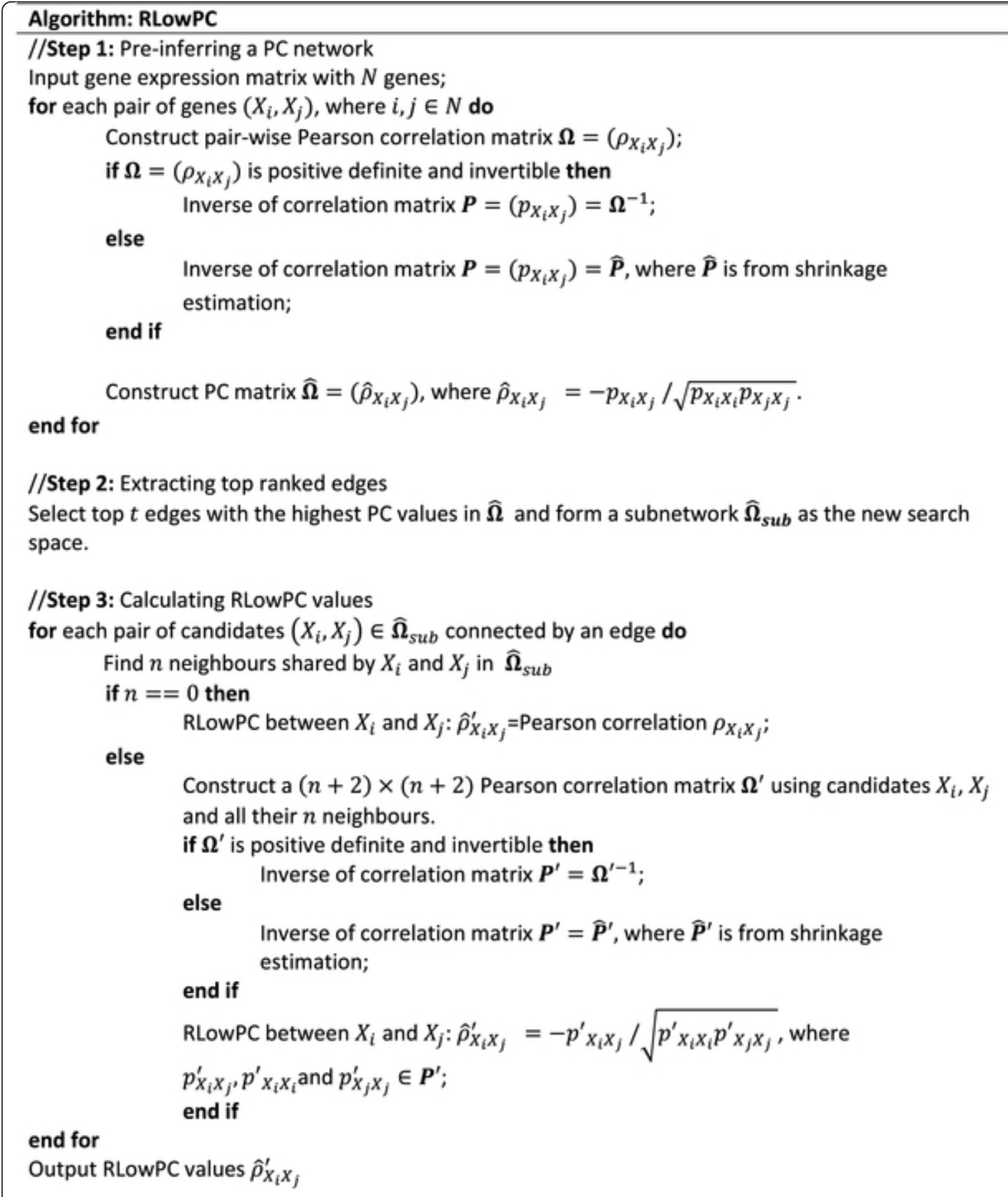


For PC and shrinkage PC calculation we have used ppcor $\mathrm{R}$ package [44] and corpcor $\mathrm{R}$ package [45], respectively.

\section{Gene expression data simulation}

The main purpose of this study is to evaluate the performance of different network inference methods on datasets that reflect real experimental setup: large number of genes in the network with limited sample sizes and perturbations. Here, to evaluate the proposed methods comprehensively, large scale gene expression datasets were generated based on a variety of network structures using GNW version 3.1 [22, 24]. We used in silico size 100 networks in DREAM4, extracted size 500 and 1000 networks from a source E.coli network with 1565 nodes and 3758 edges and size 2000 and 3000 networks from a Yeast source network with 4441 nodes and 12,873 edges as reference networks. The source networks were provided by GNW [22, 24]. The networks were denoted as GNW100, GNW500, GNW1000,
GNW2000 and GNW3000. Summaries for data generation can be found in Table 2. For each size, network extraction was repeated five times yielding five networks with different structures and kinetics for statistical analysis of the results. To generate the time-series, transcription kinetic models of reference networks were firstly generated in GNW by removing self-regulatory interactions and randomly assigning transcription factor (TF) genes to groups to produce protein binding complexes. In the time-series simulation procedure, Stochastic Differential Equations (SDEs) were used to model the transcription kinetics, gene activation by protein complexes, gene perturbations, mRNA and protein production and degradation. One-third of the genes in each time-series were randomly selected and perturbed from steady state at the initial time-point. Perturbations were implemented by varying the activation strengths in the protein binding simulations to enhance or inhibit the downstream expression of target genes. The perturbations were sustained until the

Table 2 Source network structures and synthetic datasets

\begin{tabular}{|c|c|c|c|c|c|c|c|c|}
\hline \multicolumn{2}{|c|}{ Network name } & TF-gene networks & Gene No. & Edge No. & Network density & Data generator & Data type & Ref. \\
\hline \multirow[t]{5}{*}{ GNW100 } & GNW100_1 & \multirow{5}{*}{$\begin{array}{l}\text { DREAM4 in } \\
\text { Silico size } 100\end{array}$} & 100 & 176 & 0.0356 & \multirow{25}{*}{$\begin{array}{l}\text { The TF-gene reference networks } \\
\text { were subsets of source networks } \\
\text { in GNW. In each dataset, } 1 / 3 \\
\text { genes were randomly selected } \\
\text { and perturbed. Each experiment } \\
\text { was sampled at } 21 \text { time points. } 3 \\
\text { replicates were generated by } \\
\text { adding different amount of } \\
\text { noises. The noises are simulated } \\
\text { by GNW. All the parameter } \\
\text { settings were defaults in GNW. }\end{array}$} & \multirow{25}{*}{$\begin{array}{l}\text { Time-series data } \\
\text { with multifactorial } \\
\text { perturbation }\end{array}$} & \multirow[t]{10}{*}[22,24]{} \\
\hline & GNW100_2 & & 100 & 249 & 0.0503 & & & \\
\hline & GNW100_3 & & 100 & 195 & 0.0394 & & & \\
\hline & GNW100_4 & & 100 & 211 & 0.0426 & & & \\
\hline & GNW100_5 & & 100 & 193 & 0.0390 & & & \\
\hline \multirow[t]{5}{*}{ GNW500 } & GNW500_1 & \multirow[t]{5}{*}{ E.coli } & 500 & 1365 & 0.0109 & & & \\
\hline & GNW500_2 & & 500 & 867 & 0.0069 & & & \\
\hline & GNW500_3 & & 500 & 1107 & 0.0089 & & & \\
\hline & GNW500_4 & & 500 & 947 & 0.0076 & & & \\
\hline & GNW500_5 & & 500 & 1272 & 0.0102 & & & \\
\hline \multirow[t]{5}{*}{ GNW1000 } & GNW1000_1 & \multirow[t]{5}{*}{ E.coli } & 1000 & 2337 & 0.0047 & & & \\
\hline & GNW1000_2 & & 1000 & 2455 & 0.0049 & & & \\
\hline & GNW1000_3 & & 1000 & 2089 & 0.0042 & & & \\
\hline & GNW1000_4 & & 1000 & 2171 & 0.0043 & & & \\
\hline & GNW1000_5 & & 1000 & 2249 & 0.0045 & & & \\
\hline \multirow[t]{5}{*}{ GNW2000 } & GNW2000_1 & \multirow[t]{5}{*}{ Yeast } & 2000 & 4738 & 0.0024 & & & \\
\hline & GNW2000_2 & & 2000 & 4467 & 0.0022 & & & \\
\hline & GNW2000_3 & & 2000 & 5055 & 0.0025 & & & \\
\hline & GNW2000_4 & & 2000 & 5283 & 0.0026 & & & \\
\hline & GNW2000_5 & & 2000 & 4817 & 0.0024 & & & \\
\hline \multirow[t]{5}{*}{ GNW3000 } & GNW3000_1 & \multirow[t]{5}{*}{ Yeast } & 3000 & 7515 & 0.0017 & & & \\
\hline & GNW3000_2 & & 3000 & 7998 & 0.0018 & & & \\
\hline & GNW3000_3 & & 3000 & 7626 & 0.0017 & & & \\
\hline & GNW3000_4 & & 3000 & 8075 & 0.0018 & & & \\
\hline & GNW3000_5 & & 3000 & 7333 & 0.0016 & & & \\
\hline
\end{tabular}

A number of directed network structures were generated from source networks provided by GNW. The network names, gene and edge numbers for each structure are listed in the table. Network density is defined as the true edges divided by all possible edges. The network structures were used to simulate the time-series datasets using GNW 
middle of the time-series at time point 11 when the activation strengths were changed back to initial levels. A random noise term proportional to production and degradation was introduced in the SDE model, inducing high noise for activated genes and low noise for inactivated genes. The coefficient to control the noise amplitude was set to 0.05 . Another random noise, which was independent to the noise in SDEs, was added at the final step to the expression data to simulate technical variations [46]. The parameters for activation strengths, production, degradation and noises were set as defaults in GNW. The time-series generation were repeated five times yielding five different time-series with different initial conditions and perturbations. Average results obtained from these time series as well as five different network structures are reported in this study. Parameter setting details are shown in Additional file 1: Figure S3 and Additional file 2: Configuration file for GeneNetWeaver. Three biological replicates were generated for each timeseries. By using the replicates, analysis of variance was carried out to select genes with significant expression changes across all 21 time-points with $p$-value cut-off of 0.001 . In each experiment, there are only 63 gene expression profiles generated from one perturbation used for the network construction. The repeated generation of time series data as well as the network extraction are only used for statistical purposes to take the average and calculate the variations.

\section{Evaluation of the network inference methods}

Besides the methods mentioned earlier, we also included Pearson correlation, which has been the most commonly used method to identify correlated gene pairs, as well as random guessed network, which serves a baseline for network inference performances. We also included the CLR method, which although not partial correlationbased, has been shown to perform well in several studies [30, 33, 47-49]. We divided the methods under investigation into two groups. Group one includes all the methods that deal with indirect edges explicitly, which are RLowPC, PC, PCIT, ARACNE, MRNET and MRNETB. Group two are the methods which do not deal with indirect edges explicitly and they are CLR, Pearson correlation and random guessed networks. For MI-based methods, such as ARACNE, MRNET, MRNETB and CLR networks, we have used the minet $R$ package with default parameters [50]. The MI matrices of the methods were approximated using Pearson correlation directly from continuous timeseries data $[27,49]$. The PC matrices were calculated by a shrinkage approach using corpcor $\mathrm{R}$ package [45]. The Boolean PCIT adjacency matrices were calculated using PCIT R package $[38,51]$, which was used as a weight to Pearson correlation networks [33]. For the RLowPC method, the top $(1500,2000,3000,5000,8000)$ weighted edges of inferred PC networks in GNW100, GNW500, GNW1000, GNW2000 and GNW3000 datasets were selected as search space for indirect edges. Details for tools used in the network inference analyses can be found in Table S2 in Additional file 1. In each inferred network, the top 1000 edge predictions was used to calculate True Positive (TP), False Positive (FP), True Negative (TN) and False Negative (FN) by comparing to the reference networks. The precision $(\mathrm{TP} /(\mathrm{TP}+\mathrm{FP}))$ and pAUPR (partial plot of Area Under Precision against Recall $=\mathrm{TP} /(\mathrm{TP}+\mathrm{FN}))$ values were calculated by picking the top ranked edges. pAUROC (partial Area Under the Receiver-Operating curve) was also calculated and the results were shown in the Supplementary material. All the evaluation of network inference methods was based on undirected network structures and the self-regulation edges were removed.

\section{Results}

RLowPC significantly improves the precision and recall in top predictions

Figure 2 illustrates the average pAUPR values, which are the partial Area Under Precision against Recall of the top 1000 predictions, for the different methods for different network sizes. Firstly, all methods except one case for ARACNE, outperformed the random guessed network, which proves the utility of such co-expression network analysis methods. Secondly, the performances of all methods are quite consistent across different network sizes. Within Group One, RLowPC consistently performs better than all of the other methods, with MRNET/MRNETB being the next best. Within Group two, CLR clearly outperforms the most commonly employed Pearson correlation method. The differences of pAUPR values between different methods were determined using a Student t-test in pairs between RLowPC and the other eight methods (Fig. 2). Results show that the RLowPC method is able to improve the pAUPR among the top edges significantly compared to other methods except for a few cases. The pAUROC show similar results (Additional file 1: Figure S1).

We further divided the top 1000 predictions into groups of top 1-100, 101-500 and 501-1000 (Fig. 3). The plots indicate that, once again, the precision of RLowPC method outperformed all others, regardless of which group within the top 1000 genes were selected for investigation. MRNET, MRNETB and CLR again showed slightly better performance than PC, PCIT and ARACNE and correlation methods. It is noteworthy that the precisions of all the methods are extremely low in large networks. For example, the precision median of RLowPC in the GNW3000 networks is around 0.006, which 

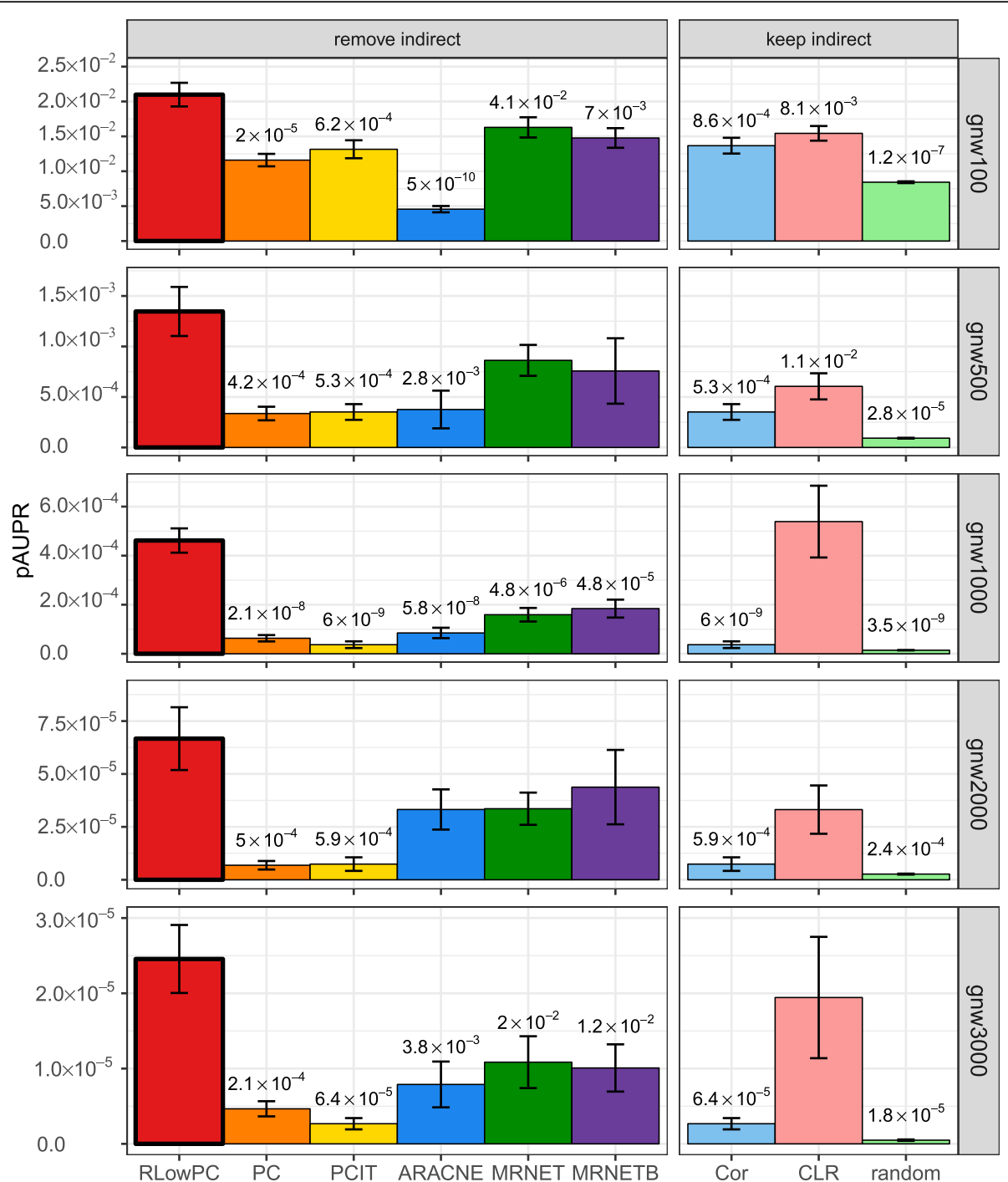

Fig. 2 Comparison of pAUPR values for different methods and different network structures. Each bar in the plots represents mean of pAUPR values from the top 1000 edge predictions. Error bars represent standard error. The differences of pAUPR values between different methods were determined using a Student t-test in pairs between RLowPC and the other eight methods. $P$-values are shown on the top of the bars if it is less than 0.05

indicates that in the top 100 predictions, only $0.6(0.6 \%)$ edges are true predictions.

\section{Clustering before network inference could improve the precision and recall in top predictions}

Given that precision and recall is very low among the top predictions for all methods for large networks, we explored whether precision can be improved by dividing the large networks into smaller highly cohesive clusters. Using the time-series data generated for GNW3000 as described above, all genes were clustered into nonoverlapping co-expressed modules using the $\mathrm{R}$ package Weighted Correlation Network Analysis (WGCNA) with default settings $[52,53]$. Then, network inference and evaluation were carried out separately and individually in each module. Essentially, WGCNA was used to break a big network into smaller non-overlapping subnetworks, at which point we carried out the network inference and evaluations within these smaller networks with the same time-series data. The pAUPR values were averaged across all the modules and it did not include genes that do not fit in any module (grey module). Similar to the simulation settings above, the clustering and evaluation procedures were repeated for five network structures, where five different time-series data were simulated for each structure. The average results were obtained. The average pAUPR values and precision distribution of the top 1000 predictions are presented in Fig. 4. Compared with the results of GNW3000 in Figs. 2 and 3, all methods evaluated have improved when the 

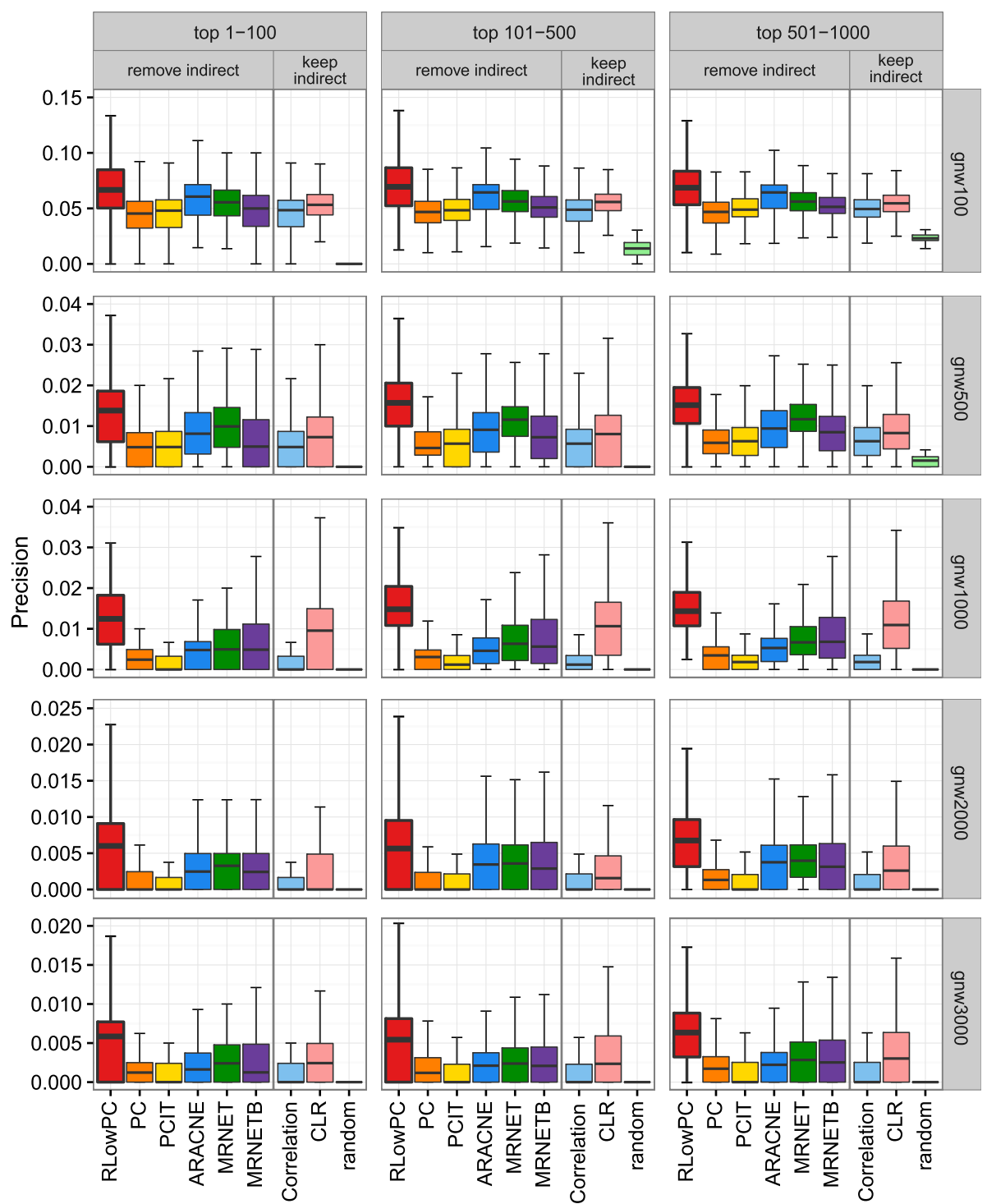

Fig. 3 Precisions within different groups of the top 1000 predicted edges. The top 1000 predicted edges are divided into three groups, top 1-100, 101-500 and 501-1000. Each bin depicts the precision distribution of the method matched to the group and the network structures

WGCNA method was used. This can be seen with the scale of average pAUPR values which increased from $1.0 \times 10^{-5}$ to $1.0 \times 10^{-3}$ (Fig. $4 \mathrm{a}$ ), while the average precision of the top 1000 predictions has changed from $3.1 \times 10^{-3}$ to $5.7 \times 10^{-3}$ when the WGCNA method is used (Fig. 4b). The pAUPR value of RLowPC method is again significantly better than PC, PCIT, ARACNE, correlation and random networks. In the groups of top 1100 and 101-500, the precision of RLowPC is better than the other eight methods and in top 501-1000 it is only better than PC, PCIT, correlation and random networks. The superior performances of RLowPC when the WGCNA method is used are also observed on the pAUROC plots (Additional file 1: Figure S2).

\section{Discussion}

The performance of different network inference methods varies according to network structures, data quantity and quality, and methodologies. The insufficiency of sampling and the high complexity of regulation kinetics prevent precise predictions of large gene regulatory networks. As a large regulatory network is often underdetermined using a small number of samples, there exists multiple plausible solutions, which cannot be distinguished by the information presented in the sample. This uncertainty in the inference of gene regulatory networks has been termed in some studies as "inferability" [54, 55]. Although our study mainly focuses on the network inference methods, special attention 

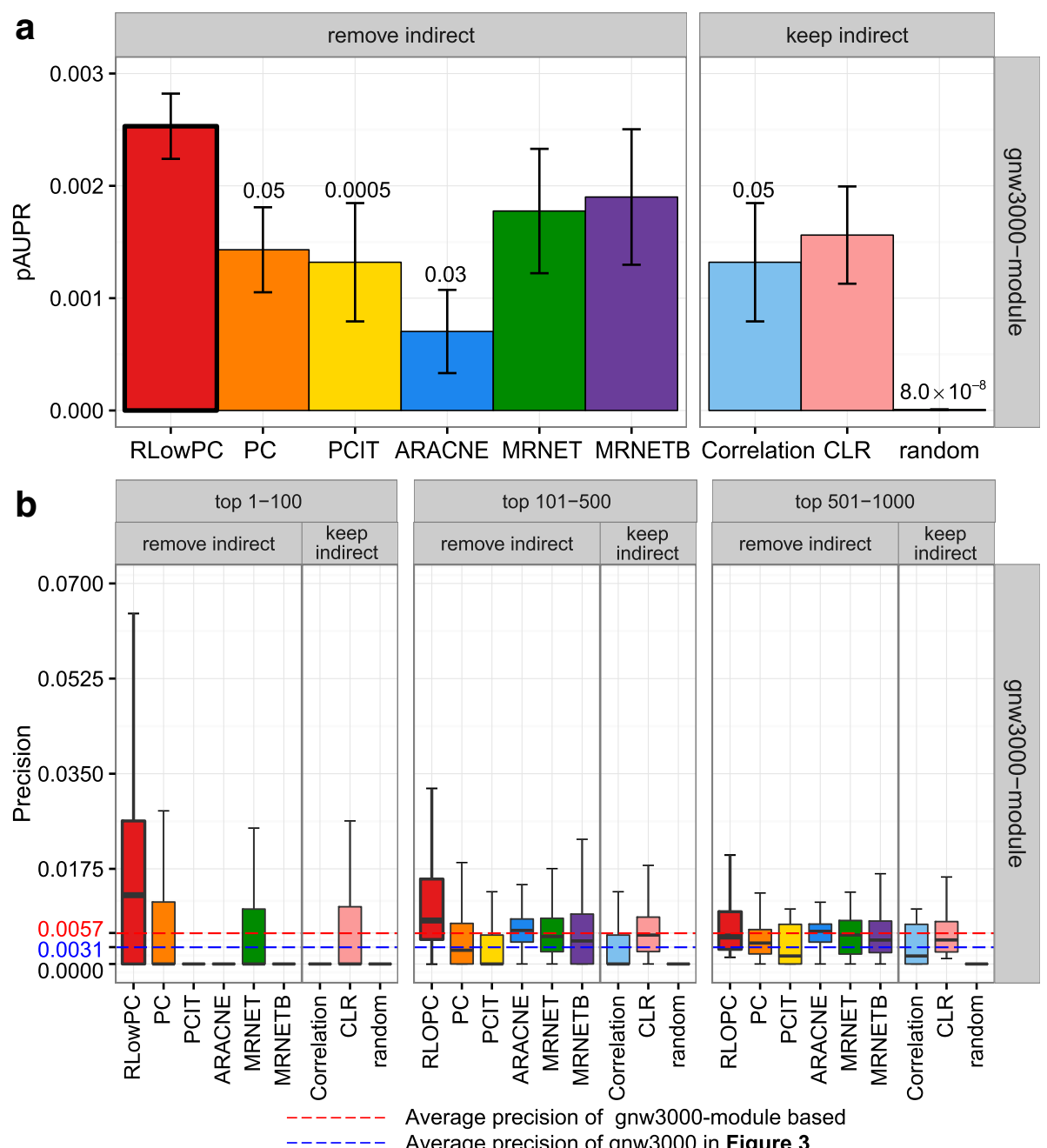

Fig. 4 Evaluation of network analysis methods within co-expression modules by WGCNA on GNW3000 networks a Barplots of average pAUPR for different methods. Error bars represent standard errors of the PAUPR values across the top 1000 predictions. A Student t-test was carried out to determine the significance of the difference of PAUPR values between RLowPC and the other eight methods. P-values are shown on the top of the bars if it is less than $0 . \mathbf{b}$ Box plots of precisions in different groups of top 1000 edge predictions. The means of precision within modules by WGCNA (0.0057) and before clustering using WGCNA (0.0031) are shown as red and blue dashed lines

should be paid to generate the most informative data when trying to construct the accurate and comprehensive underlying GRNs.

The co-expression based methods capture the relationships between genes which are perturbed directly or indirectly. Therefore, the multifactorial intervention on the regulators, as discussed in [30], or hub genes rather than on target genes will generate expression data that is more informative for regulatory inference. Results presented here are based on the time-series data corresponding to one perturbation simulation to reflect more typical experimental conditions. When there are more experiments available with different sets of genes being perturbed, the inference accuracy tends to increase with the increased number of gene expression profiles available $[35,56]$. Our data also show that the precision median increases as the experiment size increase (Fig. 5a). Using RLowPC, a precision of 0.014 is achieved in one experiment, while using PC on $10 \mathrm{ex}-$ periments only leads to a precision of 0.012 . Thus refining the top inferred edges using RLowPC is more effective in improving precision than generating data for nine more experiments.

With the number of possible edges growing factorially with increasing number of genes, the sparsity issue in large networks also becomes more prevalent. We observed that precision of the network inference methods increases with the increase of the network density (thus the decrease of network sparsity) as shown in Fig. 5b. Several types of methods have been explored to alleviate 

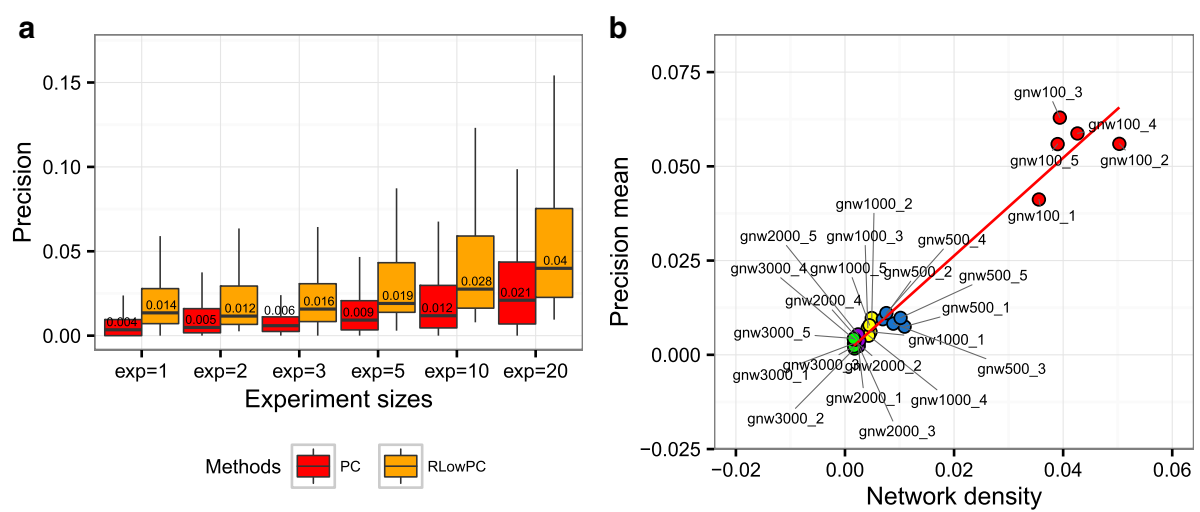

Fig. 5 Other factors that influence the precision for network inference a Boxplots on the precision of PC and RLowPC methods inferred from datasets with 1, 2, 3, 5, 10 and 20 experiments with different perturbations. b relationships between the average precisions of all network inference methods used in this study and network density. The network names shown on the plot can be found in Table 2

this problem including using network inference methods that allow imposing sparsity constraints $[31,57,58]$ or leveraging on multiple datasets on other species that are evolutionary connected [59], or incorporating prior information, such as genetic maps [60], pathways, transcription factor binding, protein-protein interactions, gene ontology, epigenetics, literature, as well as functional association databases to increase the efficiency and reduce the search space by focusing on the top weighted edges [61]. RLowPC method also uses a twostep approach to select on the high-confidence edges first. Thus there is enrichment of true regulatory relationships for the second step of the inference, which explains the improvement of gene regulatory inference performances. Similarly clustering using WGCNA also groups highly correlated and connected genes together, which we see an increase of proportion in the true regulatory relationships. This has a similar effect on the network inference performances.

AUROC and AUPR curves have been popular matrices in the evaluation of network performances [21, 30, $33,34]$. AUROC measures the area under the curve between true positive rate/recall, which is calculated as $(\mathrm{TP} /(\mathrm{TP}+\mathrm{FN}))$ and false positive rate, which is calculated as $(\mathrm{FP} /(\mathrm{FP}+\mathrm{TN})=\mathrm{FP} / \mathrm{N})$. As in big sparse networks, the negatives $(\mathrm{N})$ greatly exceed the positives (P), thus false positive rate is less discriminative when the network inference methods have very different abilities to largely reduce the false positive predictions.
In the meantime, AUPR measures the area under the curve between precision and recall. Precision, which is calculated as $(\mathrm{TP} /(\mathrm{TP}+\mathrm{FP})=1-\mathrm{FP} /(\mathrm{TP}+\mathrm{FP}))$, captures the impacts of TP or FP in the evaluation of big networks. Studies have shown that AUPR is more informative than AUROC in evaluation on datasets where the TP and TN is imbalanced. Large sparse networks are typical cases [62,63]. As the purpose of this study is to focus on the utility of co-expression network inferences methods to prioritize the novel regulatory genes pairs for experimental validation from the top ranked edges, we mainly focused on partial AUPR curve to evaluate the accuracies and power of the network inference methods on the top weighted edges, which is more relevant than using the entire area under the curve [64, 65].

One parameter required by the RLowPC method is a number to define the search space for indirect edge reduction. For large networks, a reduction space larger than the size of the top weighted edges under investigation should be applied but has to take into account the computational search space and time required. Table 3 lists the average computational time for different sizes of search space. A useful prior may be to enrich the reduction space with true gene connections. For example, cluster analysis and functional annotation using other experimental data or regulatory databases could be carried out before network inference to investigate the functions and modules of interest.

Table 3 Average computational time of different sizes of reduction space using RLowPC

\begin{tabular}{lllllllll}
\hline Top weighted edges & 1500 & 2000 & 3000 & 5000 & 8000 & 10,000 & 50,000 & 100,000 \\
\hline Time & 4.71 & 6.69 & 11.42 & 22.62 & 42.00 & 54.39 & 12.97 & 53.09 \\
Units & secs & secs & secs & secs & secs & secs & mins & mins \\
\hline
\end{tabular}

The computational time is calculated based on Dell, Windows 7, 64-bit Operating system with 16.0GB RAM and Intel(R) Core (TM) i7-4790 CPU @ 3.60GHz $3.60 \mathrm{GHz}$ processor 


\section{Conclusions}

In this paper, we present analysis of the evaluation of different regulatory network inference methods with special emphasis on large scale gene regulatory networks with limited sample size. We developed a new method, RLowPC, which improves the precision and recall in the top weighted PC network structures. We evaluated all methods on time-series datasets with only one perturbation for various sizes of networks using a small number of samples, which reflect better the high throughput gene expression data usually generated in laboratory experiments. We also demonstrated that clustering large co-expression networks into functional and informative co-expressed modules, improved the precision and recall of the regulatory inference.

\section{Additional files}

Additional file 1: File contains additional Figures and Tables. Figure S1. Bar plots of pAUROC values for top 1000 edge predictions. Figure S2. Bar plots of PAUROC values of top 1000 predictions for GNW3000 modulebased. Figure S3. GNW settings for data simulation. Figure S4. Examples of evaluation results. Table S1. Summaries of evaluation of gene network inference methods. Table S2. R packages used to construct and evaluate GRNs. (DOCX $1867 \mathrm{~kb}$ )

Additional file 2: Configuration file for GeneNetWeaver (GNW). The settings in the file were load in GNW to generate synthetic data. (DOCX $28 \mathrm{~kb}$ )

\section{Abbreviations}

ARACNE: Algorithm for the reconstruction of accurate cellular networks; AUROC: Area under the receiver-operating characteristic curve; AUPR: Area under the precision recall curve; BN: Bayesian network; CLR: Context likelihood or relatedness; DREAM: Dialogue for reverse engineering assessments and methods; FN: False negative; FP: False positive; GCN: Gene co-expression network; GENIE3: Gene network inference with ensemble of trees; GNW: GeneNetWeaver; GRN: Gene regulatory network; MI: Mutual information; MRNET: Minimum redundancy networks; MRNETB: Minimum redundancy networks using backward elimination; pAUROC: Partial area under the receiver-operating characteristic curve; PAUPR: Partial area under the precision-recall curve; PC: Partial correlation; PCIT: Partial correlation coefficient with information theory; RN: Relevance network; RLowPC: Relevance low order partial correlation; SDEs: Stochastic differential eqs.; TN: True negative; TP: True positive; WGCNA: Weighted correlation network analysis

\section{Acknowledgements}

We would like to thank Dr. Katherine Denby (University of York) for suggestions on gene network construction and lain Milne (James Hutton Institute) for technical assistance.

\section{Funding}

This project was supported by joint PhD studentship Program from the James Hutton Institute and the University of Dundee [to W.G] and the Scottish Government Rural and Environment Science and Analytical Services division (RESAS) [to J.B., R.W. and R.Z.].

\section{Availability of data and materials}

Project name: RLowPC

Project home page: https://github.com/wyguo/RLowPC

Version: 0.1

Operating system(s): Platform independent

Programming language: $\mathrm{R}$ (version 3.2.3)

Other requirements: corpcor, ggplot2, minet, PCIT, ppcor and WGCNA R packages.

Licence: GPL-3
Synthetic data are available upon request. Please contact corresponding author Runxuan Zhang: Runxuan.zhang@hutton.ac.uk

\section{Author's contributions}

$R Z$ and WG defined the project and design the simulation experiments. WG carried out the simulations and analyses. WG, RZ, JB and CC wrote the manuscript. All the authors engaged in discussions to improve the project and made contributions to improve the final version of manuscript. All authors read and approved the final manuscript.

\section{Competing interests}

The authors declare that they have no competing interests.

\section{Author details}

${ }^{1}$ Information and Computational Sciences, The James Hutton Institute, Invergowrie, Dundee, Scotland DD2 5DA, UK. ²Plant Sciences Division, School of Life Sciences, University of Dundee, Invergowrie, Dundee, Scotland DD2 5DA, UK. ${ }^{3}$ Division of Mathematics, University of Dundee, Nethergate, Dundee, Scotland DD1 4HN, UK. ${ }^{4}$ Cell and Molecular Sciences, The James Hutton Institute, Invergowrie, Dundee, Scotland DD2 5DA, UK.

Received: 4 November 2016 Accepted: 9 June 2017

Published online: 19 June 2017

References

1. Friedman N, Linial M, Nachman I, Pe'er D. Using Bayesian networks to analyze expression data. J Comput Biol. 2000;7(3-4):601-20.

2. Markowetz F, Spang R. Inferring cellular networks-a review. BMC Bioinformatics. 2007;8(Suppl 6):S5

3. Murphy KP. Dynamic Bayesian networks: representation, inference and learning. Berkeley: University of California; 2002.

4. Perrin B-E, Ralaivola L, Mazurie A, Bottani S, Mallet J, d'Alche-Buc F. Gene networks inference using dynamic Bayesian networks. Bioinformatics. 2003;19:li138-48.

5. Lahdesmaki H, Hautaniemi S, Shmulevich I, Yli-Harja O. Relationships between probabilistic Boolean networks and dynamic Bayesian networks as models of gene regulatory networks. Signal Process. 2006;86(4):814-34.

6. Hache $H$, Lehrach $H$, Herwig R. Reverse engineering of gene regulatory networks: a comparative study. EURASIP J Bioinformatics Syst Biol. 2009; 2009:1-12.

7. Bornholdt S. Boolean network models of cellular regulation: prospects and limitations. J R Soc Interface. 2008;5(Suppl 1):S85-94.

8. Martin S, Zhang Z, Martino A, Faulon JL. Boolean dynamics of genetic regulatory networks inferred from microarray time series data. Bioinformatics. 2007;23(7):866-74.

9. de Jong $\mathrm{H}$. Modeling and simulation of genetic regulatory systems: a literature review. J Comput Biol. 2002;9(1):67-103.

10. Linde J, Schulze S, Henkel SG, Guthke R. Data- and knowledge-based modeling of gene regulatory networks: an update. EXCLI J. 2015;14:346-78.

11. Bansal M, Belcastro V, Ambesi-Impiombato A, di Bernardo D: How to infer gene networks from expression profiles. Mol Syst Biol 2007, 3(1): 78.

12. Werhli AV, Grzegorczyk M, Husmeier D. Comparative evaluation of reverse engineering gene regulatory networks with relevance networks, graphical gaussian models and bayesian networks. Bioinformatics. 2006;22(20):2523-31.

13. Zhang B, Horvath S. A general framework for weighted gene co-expression network analysis. Stat Appl Genet Mol Biol. 2005:4:1128. Article17

14. Friedman N. Inferring cellular networks using probabilistic graphical models. Science. 2004;303(5659):799-805.

15. Song $L$, Langfelder $P$, Horvath S. Comparison of co-expression measures: mutual information, correlation, and model based indices. BMC Bioinformatics. 2012;13:328.

16. Roy S, Bhattacharyya DK, Kalita JK. Reconstruction of gene co-expression network from microarray data using local expression patterns. BMC Bioinformatics. 2014;15(7):1-14.

17. Ballouz S, Verleyen W, Gillis J. Guidance for RNA-seq co-expression network construction and analysis: safety in numbers. Bioinformatics. 2015;31(13):2123-30.

18. Kogelman LJ, Cirera S, Zhernakova DV, Fredholm M, Franke L, Kadarmideen $\mathrm{HN}$. Identification of co-expression gene networks, regulatory genes and pathways for obesity based on adipose tissue RNA sequencing in a porcine model. BMC Med Genet. 2014;7:57. 
19. DiLeo MV, Strahan GD, den Bakker M, Hoekenga OA. Weighted correlation network analysis (WGCNA) applied to the tomato fruit metabolome. PLoS One. 2011;6(10):e26683.

20. Prill RJ, Marbach D, Saez-Rodriguez J, Sorger PK, Alexopoulos LG, Xue X, et al. Towards a rigorous assessment of systems biology models: the DREAM3 challenges. PLoS One. 2010;5(2):e9202

21. Marbach D, Prill RJ, Schaffter T, Mattiussi C, Floreano D, Stolovitzky G. Revealing strengths and weaknesses of methods for gene network inference. Proc Natl Acad Sci. 2010;107(14):6286-91.

22. Marbach D, Schaffter T, Mattiussi C, Floreano D. Generating Realistic In Silico Gene Networks for Performance Assessment of Reverse Engineering Methods. J. Comput. Biol. 2009;16:229-39.

23. Yip KY, Alexander RP, Yan KK, Gerstein M. Improved reconstruction of in silico gene regulatory networks by integrating knockout and perturbation data. PLoS One. 2010:5(1):e8121.

24. Schaffter T, Marbach D, Floreano D. GeneNetWeaver: in silico benchmark generation and performance profiling of network inference methods. Bioinformatics. 2011;27(16):2263-70.

25. Young WC, Raftery AE, Yeung KY. Fast Bayesian inference for gene regulatory networks using ScanBMA. BMC Syst Biol. 2014;8:47.

26. Huynh-Thu V, Irrthum A, Wehenkel L, Geurts P. Inferring regulatory networks from expression data using tree-based methods. PLoS One. 2010;5(9):e12776.

27. Meyer P, Marbach D, Roy S, Kellis M. Information-theoretic inference of gene networks using backward elimination. In: BIOCOMP, International Conference on Bioinformatics and Computational Biology: 2010;700-5

28. Margolin AA, Nemenman I, Basso K, Wiggins C, Stolovitzky G, Dalla Favera R, et al. ARACNE: an algorithm for the reconstruction of gene regulatory networks in a mammalian cellular context. BMC Bioinformatics. 2006; 7(Suppl 1):S7.

29. Meyer PE, Kontos K, Lafitte F, Bontempi G. Information-theoretic inference of large transcriptional regulatory networks. EURASIP J Bioinform Syst Biol. 2007:79879

30. Marbach D, Costello JC, Kuffner R, Vega NM, Prill RJ, Camacho DM, et al. Wisdom of crowds for robust gene network inference. Nat Methods. 2012;9(8):796-804

31. Rogers S, Girolami M. A Bayesian regression approach to the inference of regulatory networks from gene expression data. Bioinformatics. 2005;21(14):3131-7.

32. Van den Bulcke T, Van Leemput $K$, Naudts $B$, van Remortel $P, M a H$, Verschoren A, et al. SynTReN: a generator of synthetic gene expression data for design and analysis of structure learning algorithms. BMC Bioinformatics. 2006;7:43.

33. Bellot $P$, Olsen $C$, Salembier $P$, Oliveras-Verges A, Meyer PE. NetBenchmark: a bioconductor package for reproducible benchmarks of gene regulatory network inference. BMC Bioinformatics. 2015;16:312.

34. Allen JD, Xie Y, Chen M, Girard L, Xiao G. Comparing statistical methods for constructing large scale gene networks. PLoS One. 2012;7(1):e29348.

35. Steinke F, Seeger M, Tsuda K. Experimental design for efficient identification of gene regulatory networks using sparse Bayesian models. BMC Syst Biol. 2007;1(1):51

36. Dehghannasiri R, Yoon BJ, Dougherty ER. Efficient experimental design for uncertainty reduction in gene regulatory networks. BMC Bioinformatics. 2015;16(Suppl 13):S2

37. Cover TM, Thomas JA: Elements of information theory: Wiley-Interscience; 2006.

38. Reverter A, Chan EK. Combining partial correlation and an information theory approach to the reversed engineering of gene co-expression networks. Bioinformatics. 2008;24(21):2491-7.

39. Peng $\mathrm{H}$, Long $\mathrm{F}$, Ding $\mathrm{C}$. Feature selection based on mutual information: criteria of max-dependency, max-relevance, and min-redundancy. IEEE Trans Pattern Anal Mach Intell. 2005;27(8):1226-38.

40. Barabasi AL, Oltvai ZN. Network biology: understanding the cell's functional organization. Nat Rev Genet. 2004;5(2):101-13.

41. Albert R. Scale-free networks in cell biology. J Cell Sci. 2005;118(Pt 21): 4947-57.

42. de la Fuente A, Bing N, Hoeschele I, Mendes P. Discovery of meaningful associations in genomic data using partial correlation coefficients. Bioinformatics. 2004;20(18):3565-74.

43. Zuo Y, Yu G, Tadesse MG, Ressom HW. Biological network inference using low order partial correlation. Methods (San Diego, Calif). 2014;69(3):266-73.
44. Kim S. Ppcor: an R package for a fast calculation to semi-partial correlation coefficients. Commun Stat Appl Methods. 2015:22(6):665-74.

45. Schäfer J, Strimmer K. A shrinkage approach to large-scale covariance matrix estimation and implications for functional genomics. Stat Appl Genet Mol Biol. 2005;4(1):32.

46. Tu Y, Stolovitzky G, Klein U. Quantitative noise analysis for gene expression microarray experiments. Proc Natl Acad Sci U S A. 2002;99(22):14031-6.

47. Madhamshettiwar PB, Maetschke SR, Davis MJ, Reverter A, Ragan MA. Gene regulatory network inference: evaluation and application to ovarian cancer allows the prioritization of drug targets. Genome Med. 2012;4(5):41.

48. Faith JJ, Hayete B, Thaden JT, Mogno I, Wierzbowski J, Cottarel G, et al. Large-scale mapping and validation of Escherichia coli transcriptional regulation from a compendium of expression profiles. PLoS Biol. 2007;5(1):e8.

49. Olsen C, Meyer PE, Bontempi G. On the impact of entropy estimation on transcriptional regulatory network inference based on mutual information. EURASIP J Bioinform Syst Biol. 2009;1:308959.

50. Meyer PE, Lafitte F, Bontempi G: minet: A R/Bioconductor package for inferring large transcriptional networks using mutual information. BMC Bioinformatics 2008, 9:461

51. Watson-Haigh NS, Kadarmideen HN, Reverter A. PCIT: an R package for weighted gene co-expression networks based on partial correlation and information theory approaches. Bioinformatics. 2010;26(3):411-3.

52. Langfelder $P$, Horvath S. WGCNA: an R package for weighted correlation network analysis. BMC Bioinformatics. 2008;9:559.

53. Zhao W, Langfelder P, Fuller T, Dong J, Li A, Hovarth S. Weighted gene coexpression network analysis: state of the art. J Biopharm Stat. 2010;20(2):281-300

54. Ud-Dean SM, Gunawan R. Ensemble inference and inferability of gene regulatory networks. PLoS One. 2014;9(8):e103812.

55. Ud-Dean SM, Heise S, Klamt S, Gunawan R. TRaCE+: ensemble inference of gene regulatory networks from transcriptional expression profiles of gene knock-out experiments. BMC Bioinformatics. 2016;17:252.

56. Altay G. Empirically determining the sample size for large-scale gene network inference algorithms. IET Syst Biol. 2012;6(2):35-43.

57. Slavov N: Inference of Sparse Networks with Unobserved Variables. Application to Gene Regulatory Networks. In: Proceedings of the Thirteenth International Conference on Artificial Intelligence and Statistics; Proceedings of Machine Learning Research: Edited by Yee Whye TMike T. PMLR 2010: 757-764

58. Sarder P, Schierding W, Cobb JP, Nehorai A. Estimating sparse Gene regulatory networks using a Bayesian linear regression. IEEE Transactions on NanoBioscience. 2010;9(2):121-31.

59. Omranian N, Eloundou-Mbebi JMO, Mueller-Roeber B, Nikoloski Z. Gene regulatory network inference using fused LASSO on multiple data sets. Sci Rep. 2016;6:20533

60. Flassig RJ, Heise S, Sundmacher K, Klamt S. An effective framework for reconstructing gene regulatory networks from genetical genomics data. Bioinformatics. 2013:29(2):246-54

61. Studham ME, Tjärnberg A, Nordling TEM, Nelander S, Sonnhammer ELL. Functional association networks as priors for gene regulatory network inference. Bioinformatics. 2014;30(12):i130-8.

62. Saito $T$, Rehmsmeier M. The precision-recall plot is more informative than the ROC plot when evaluating binary classifiers on imbalanced datasets. PLoS One. 2015;10(3):e0118432.

63. Davis J, Goadrich M: The Relationship Between Precision-Recall and ROC Curves. In ICML '06: Proceedings of the 23rd international conference on Machine learning 2006:233-240.

64. Ma H, Bandos Al, Rockette HE, Gur D. On use of partial area under the ROC curve for evaluation of diagnostic performance. Stat Med. 2013; 32(20):3449-58.

65. Walter SD. The partial area under the summary ROC curve. Stat Med. 2005; 24(13):2025-40 